\title{
Mit einheitlicher Finanzierung die Prämien entlasten!
}

\author{
Jürg Schlup \\ Dr. med., Präsident der FMH
}

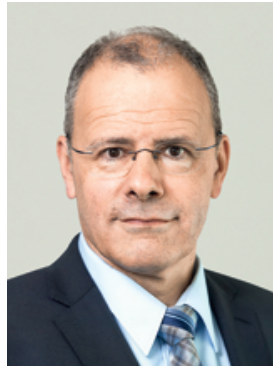

Die steigenden Krankenkassenprämien sind ein Dauerthema. Dass der wahrgenommene Handlungsdruck in der Politik aber eine neue Qualität erreicht, zeigen die aktuellen parlamentarischen Forderungen nach Staatstarifen und Globalbudgets. ${ }^{1}$ Dieses ernsthafte Erwägen einer Rationierung in der ambulanten Versorgung ist vor allem wegen der ungünstigen Auswirkungen auf Patientenversorgung und Kostenentwicklung unverständlich. Darüber hinaus irritiert auch, dass die Politik gleichzeitig das grösste Hindernis für Effizienzgewinne seit Jahren ungelöst vor sich herschiebt: die unterschiedliche Finanzierung ambulanter und stationärer Leistungen. Lassen Sie mich ein paar Fakten aufführen, die nachdenklich stimmen sollten:

Die einheitliche Finanzierung dämpft den Prämienanstieg, der durch die Verlagerung stationärer Leistungen in den ambulanten Bereich entsteht.

- Krankenkassenprämien spiegeln nicht die Gesundheitskosten wider: Alljährlich hören wir, dass die Gesundheitsausgaben wachsen und folglich auch die Prämien steigen müssten. Dabei fallen entscheidende Details meist unter den Tisch: Nur 37\% der Gesundheitsausgaben werden über Prämien finanziert. Und: Die Gesundheitsausgaben sind seit 1996 um 72\% gestiegen, die Prämien hingegen um $107 \%{ }^{3}$, also deutlich stärker! Wie kann das sein?

Siehe Motion 16.3987 und parl. Initiativen 17.401 und 17.402; Ausführungen dazu in: Schlup J. Falsche Lösungsvorschläge für ein ernstzunehmendes Problem, Schweiz Ärztezeitung. 2017;98(9)271.

2 Kosten und Finanzierung des Gesundheitswesens nach Leistungserbringern und Finanzierungsregimes. BfS Neuchâtel. 27.10.2016.

3 Gesundheitswesen Schweiz. Ausgabe 2017 Interpharma: Basel

4 PwC. Ambulant vor stationär. Oder wie sich eine Milliarde jährlich einsparen lässt. www.pwc.ch/ gesundheitswesen
- Einsparungen durch konsequentes «ambulant vor stationär» führen zu Prämienerhöhungen: Ambulante Behandlungen werden bisher zu 100\% über Prämien finanziert, stationäre Behandlungen aber mehrheitlich über Steuern. Wenn nun, bedingt durch den medizinischen Fortschritt, stationäre Behandlungen ambulant durchgeführt werden, entfällt die Steuersubvention. Die Folgen: Die Prämien steigen - obwohl die Behandlungskosten reduziert werden! Damit erhöht sich auch der Anteil der Gesundheitsausgaben, der über die Krankenkassen-

- Der ambulante Bereich ist Kostendämpfer - nicht Kostentreiber: Ambulante Versorgung ist eine sehr Kopfprämien finanziert wird. kostengünstige Behandlungsform. Wer den Kostenanstieg im Gesundheitswesen mildern möchte, muss die ambulante Versorgung darum ausbauen nicht einschränken! Eine Analyse von PwC Schweiz ${ }^{4}$ zeigte vor kurzem, dass die ungenutzten ambulanten Möglichkeiten ein Sparpotential von jährlich einer Milliarde Franken bergen. Dies zeigt deutlich, dass Kostensteigerungen im ambulanten Bereich nicht selten ungleich grössere Einsparungen an anderen Orten gegenüberstehen.

- Die Fehlanreize unseres Finanzierungssystems verhindern das Realisieren von Sparpotential. Beispiel: Eine Krankenversicherung, die konsequent auf «ambulant vor stationär» setzt, indem sie z.B. stationäre Kostengutsprachen besonders kritisch prüft, würde den Kantonen zwar viel Geld sparen, müsste die deswegen zunehmenden ambulanten Behandlungen aber vollständig alleine finanzieren. Folglich müsste sie die Prämien erhöhen und würde Versicherte an die Konkurrenz verlieren. Die mit einer Reduktion von stationären Behandlungen verbundenen Einsparungen können heute kaum an den Prämienzahler weitergegeben werden.

Die ungenutzten ambulanten Möglichkeiten bergen ein Effizienzpotential von einer Milliarde Franken pro Jahr.

In unserem Gesundheitssystem werden Sparpotentiale nicht realisiert, weil der Nutzen nicht bei demjenigen anfällt, der den Aufwand dafür betreibt - oder der Einsatz für Effizienzgewinne sogar deutliche Nachteile bringt. Bei einer einheitlichen Finanzierung ambulanter und stationärer Leistungen stünde hingegen das gemeinsame Interesse möglichst geringer Gesamtkosten im Vordergrund. Die Umstellung wäre einfach, wenn ein konstanter Finanzierungsanteil der Kantone neu über eine gemeinsame Einrichtung einfliessen würde. Die kantonale Versorgungsverantwortung bliebe gewahrt. Bevor Staatstarife und rationierende Globalbudgets diskutiert werden, gilt es dieses prämienentlastende und ohne Versorgungseinschränkungen realisierbare Sparpotential auszuschöpfen. 